\title{
Searching for Standard Model adjoint scalars with diboson resonance signatures
}

\author{
Linda M. Carpenter and Russell Colburn \\ Department of Physics, The Ohio State University, \\ 191 W. Woodruff Ave, Columbus, OH, 43210 U.S.A. \\ E-mail: lmc@physics.osu.edu, colburn.36@osu.edu
}

ABSTRACT: We explore the phenomenology of scalar fields in the adjoint representation of SM gauge groups. We write a general set of dimension 5 effective operators in which SM adjoint scalars couple to pairs of standard model bosons. Using these effective operators, we explore new possible decay channels of a scalar color octet into a gluon and a $\mathrm{Z}$ boson, another gluon, or a photon. We recast several analyses from Run I of the LHC to find constraints on an a scalar octet decaying into these channels, and we project the discovery potential of color octets in our gluon+photon channel for the $14 \mathrm{TeV}$ run of LHC.

Keywords: Exotics, Hadron-Hadron scattering, Beyond Standard Model, Particle and resonance production

ARXIV EPRINT: 1509.07869 


\section{Contents}

1 Introduction 1

$\begin{array}{llr}2 & \text { Effective operators } & 2\end{array}$

3 High energy models $\quad 3$

4 Production and decay of scalar adjoints $\quad 6$

5 Current limits $\quad 8$

5.1 The jet $+\gamma$ channel $\quad 9$

$\begin{array}{lll}5.2 & \text { Dijet channel } & 10\end{array}$

$\begin{array}{ll}5.3 \text { The monojet channel } & 12\end{array}$

$\begin{array}{lll}5.4 & \text { Heavy boson plus jet channel } & 14\end{array}$

$\begin{array}{ll}5.5 \text { Combined results } & 14\end{array}$

6 Projection for LHC 14 16

$\begin{array}{lll}7 & \text { Conclusions } & 17\end{array}$

$\begin{array}{lr}\text { A Additional color-adjoint operators } & 18\end{array}$

\section{Introduction}

With Run II of the LHC now in progress, we are now able to probe well into the TeV range in the search for new particles. One discoverable class of heavy particles which appears in many Beyond the Standard Model (BSM) scenarios is that of scalar fields in adjoint representations of the Standard Model (SM) gauge groups. Scenarios with scalar adjoints are quite diverse and candidate fields include chiral states that mix with gauginos in Rsymmetric extensions of the Minimal Supersymmetric Standard Model [1, 2], triplet Higgs fields, various states in Minimal Flavor Violating models [3], and KK partners of gauge bosons in extra-dimensional models [4]. Proposed search channels for these states vary depending on the scenario, but include such signatures as jets plus missing energy, $t \bar{t}$ and dijet resonances, and searches for $\mathrm{W} / \mathrm{Z}+$ jets [5-9].

In this work we will explore the consequences of couplings between scalar adjoint fields and pairs of SM gauge bosons. We will begin in the framework of Effective Operator analysis. As we have seen from recent studies in Higgs physics and Dark Matter phenomenology, this is an extremely useful tool for systematically cataloging all possible interactions consistent with symmetries $[10,11]$. We first present the most general effective Lagrangian up to dimension 5 in which scalar fields in adjoint representations of the SM gauge groups couple 
to pairs of gauge bosons. These operators are allowed by all symmetries of the theory, we will therefore assume they are generically non-zero. Some dimension 5 operators involving scalar adjoints have been well studied, for example the dimension 5 coupling between a scalar color octet and two gluons. Here, we will present several additional operators which induce new diboson couplings.

We also discuss possible UV completions of the effective theory in several scenarios. These completions will involve integrating out a messenger sector of fields which are charged under multiple SM gauge groups. We will consider both supersymmetric and non-supersymmetric completions of the effective operators. We will see that for typical completions, for example simple messenger sectors consisting of vector-like fermions, gauge invariance demands the simultaneous existence of multiple dimension 5 operators. In general individual operators cannot be arbitrarily ignored. We then study the collider phenomenology induced by the operators in our effective Lagrangian.

We choose to explore the phenomenology of a scalar color octet whose production and decay proceeds through loop-level couplings to pairs of Standard Model bosons. In particular we focus on the effective couplings between the scalar octet and a gluon and photon/Z-boson. We argue that new proposed decay channels for the octet opens a window to constrain or discover various models of octet scalars. We recast several $8 \mathrm{TeV} \mathrm{LHC}$ analyses to find current constraints on effective operators coefficients. These analyses include searches for dijet resonances, searches for excited quarks, and the inclusive search in the monojet channel. We then present the discovery potential for the scalar octet in a jet plus photon channel for the $14 \mathrm{TeV}$ run of LHC.

This paper proceeds as follows, in section 2 we consider effective operators where a scalar SM adjoint may couple to gauge bosons. In section 3 we discuss possible UV completions of the effective Lagrangian. In section 4 we focus on the production and decay modes of a scalar color octet at LHC given our set of effective operators. In section 5 we present the bounds on scalar color adjoints in our scenario coming from a recast of existing searches LHC Run 1. In section 6 we present a sensitivity search for scalar adjoints in $S \rightarrow g \gamma$ channel in the $14 \mathrm{TeV}$ run of LHC. Section 7 concludes.

\section{Effective operators}

We will now explore the couplings of SM adjoint scalars to pairs of gauge bosons, beginning with operators of the lowest dimension. We consider two new spin 0 adjoint fields, color octet, $\mathrm{S}$, with quantum numbers $(8,1)$ under SM gauge groups, and a weak triplet $\mathrm{T}$ with quantum numbers $(1,3)$ under SM gauge groups. At dimension 4, pairs of scalar adjoints have couplings to pairs of gauge bosons through their kinetic terms. However, couplings between a single adjoint and a pair of gauge bosons must come at higher dimension. We will now consider the complete set of gauge and Lorentz invariant operators of dimension 5 where a scalar adjoint couples to pairs of gauge bosons.

For the scalar color octet the gauge and Lorentz invariant dimension 5 operators are

$$
L=\frac{d^{a b c}}{\Lambda_{2}} S_{a} G_{b}^{\mu \nu} G_{c, \mu \nu}+\frac{1}{\Lambda_{1}} S_{a} G^{a, \mu \nu} B_{\mu \nu}
$$


where $\Lambda_{i}$ is the scale of new physics at which the operator is generated. Here, $G^{\mu \nu}$ is the $\mathrm{SU}(3)$ field strength tensor and $B_{\mu \nu}$ is the U(1) field strength tensor. The first operator couples the color adjoint to a pair of gluons, and is familiar from previous studies of color adjoint phenomenology. In this operator, the color indices of the gluons and adjoint scalar are contracted symmetrically with $d^{a b c}$. The second operator is also gauge and Lorentz invariant. In this operator, the Lorentz indices are contracted between field strength tensors, while the color index is contracted between the gluon and the color octet. This is an effective coupling between the scalar octet, one gluon, and a photon/Z-boson.

Similarly we may consider a similar operator governing the $\mathrm{SU}(2)$ adjoint $\mathrm{T}$

$$
L=\frac{1}{\Lambda_{T W B}} T_{i} W_{i}^{\mu \nu} B_{\mu \nu}
$$

where $W^{\mu \nu}$ is the $\mathrm{SU}(2)$ field strength tensor, and $B_{\mu \nu}$ is the $\mathrm{U}(1)$ field strength tensor. Lorentz indices are contracted between the field strength tensors, while the $\mathrm{SU}(2)$ indices are contracted between the adjoint $\mathrm{T}$ and the $\mathrm{SU}(2)$ field strength tensor. Unlike $\mathrm{SU}(3)$, $\mathrm{SU}(2)$ does not have a factor $d_{a b c}$, therefore we may not write a coupling between the field $\mathrm{T}$ and two $W^{\mu \nu}$ 's as above. However, we may extend the list of possible gauge invariant operators if we allow the insertion of Higgs fields to soak up SU(2) indices. These operators will be of dimension 7, but once Higgs vevs are inserted, they become operators of effective dimension 5 . These dimension 7 operators are

$$
\begin{aligned}
L= & \frac{1}{\Lambda_{T B B}^{3}}\left[H^{\dagger} T H\right] B^{\mu \nu} B_{\mu \nu}+\frac{1}{\Lambda_{T W W^{3}}}\left[H^{\dagger} T H\right] W^{\mu \nu} W_{\mu \nu}+\frac{1}{\Lambda_{T G G}^{3}}\left[H^{\dagger} T H\right] G^{\mu \nu} G_{\mu \nu} \\
& +\frac{1}{\Lambda_{S G W}^{3}} S^{a} G_{a}^{\mu \nu}\left[H^{\dagger} W_{\mu \nu}^{i} H\right] .
\end{aligned}
$$

In the first three terms we have contracted the $\mathrm{SU}(2)$ indices of the adjoint $\mathrm{T}$ with those of the Higgs fields to make an $\mathrm{SU}(2)$ singlet; this is indicated by brackets. In the last term, $\mathrm{SU}(2)$ indicies are contracted between the $\mathrm{SU}(2)$ field strength tensor and the Higgs doublets. The $\mathrm{SU}(2)$ structure of these operators is quite reminiscent of loop induced operators in two Higgs doublet scenarios which couple the Higgs fields to SM field strength tensors as, for example, in references $[12,13]$. The operators above are of dimension 7 , however once the Higgs vev is inserted one get operators of effective dimension 5, which are suppressed by two powers of $\frac{v}{\Lambda}$. In the appendix we make a more complete list of operators for more complicated models where the scalar color octet may have additional quantum numbers under the other SM gauge groups. We expect the operators above may be generated at loop level by various UV completions of the theory. We give some illustrative examples in the next section.

\section{High energy models}

SM scalar adjoints appear in various BSM scenarios. Here we are considering models in which the dominant decay of these states is to pairs of SM gauge bosons. There are a variety of frameworks in which these operators may be completed. 

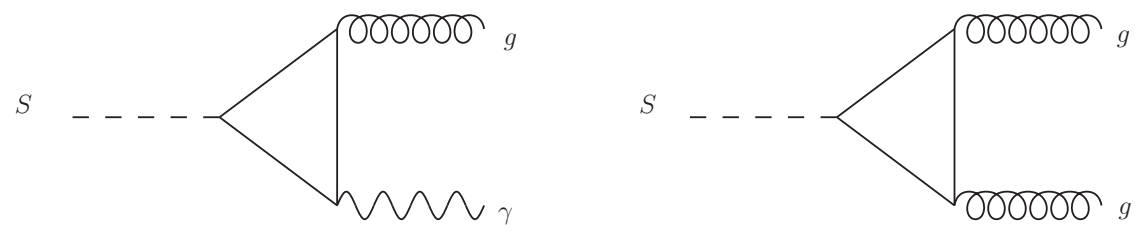

Figure 1. Decay through mediators to diboson final states.

One scenario that naturally lends itself this type of phenomenology may be models of extended technicolor. Such models typically contain SM adjoint scalar which are bound states of techni quarks/leptons that are charged under the extended technicolor gauge group in addition to SM gauge groups. Often the scalar adjoints are the lightest states in the theory. Effective operators like those in equations (2.1) and (2.2) couple the techni-adjoint to pairs of SM gauge bosons, see for example [14, 15]. It is a model building question as to which these couplings dominate over couplings between the adjoints and pairs of SM fermions. However the simplest realizations of such models are currently disfavoured by electroweak fit constraints and other theoretical challenges.

A very simple and calculable realization of our effective operators is one where they are produced by integrating out heavy fields which have quantum numbers under several SM gauge groups. One of the simplest completions may be to include one or more generations of heavy vector-like quarks or leptons which carry hypercharge in addition to $\mathrm{SU}(2)$ or $\mathrm{SU}(3)$ quantum numbers. Sets of these vector-like "messenger" fields may couple to our scalar adjoint fields through Yukawa-like terms. For example, a set of vector-like quarks which carry hypercharge have quantum numbers $(3,1)_{Y}$ and may couple to a scalar octet to generate the Feynmann diagrams in figure 1. These diagrams correspond to the operators in equation (2.1). The exact ratio of the operator coefficients $\Lambda_{2}$ and $\Lambda_{1}$ will depend on the hypercharge of the messengers. A general estimate for the ratio of effective couplings might be $g_{3} / g_{1}$, though this depends on the details of messenger sector. The effective coupling to $\mathrm{U}(1)$ gauge bosons is non-negligible and will provide additional production and decay channels for a scalar octet. This opens new windows for collider searches to illuminate new physics sectors.

One large class of models which contain SM adjoint scalars are supersymmetric theories in which gauginos are Dirac as opposed to Majorana [1, 2]. In Dirac gaugino models, gauginos get mass by mixing with new chiral fields which are adjoints under the Standard Model gauge groups. In order to generate the Dirac Mass the relevant super-potential operator is

$$
W=\int d^{2} \theta \frac{W_{\alpha}^{\prime} W^{\alpha} A}{\Lambda}=\frac{D \lambda \psi_{A}}{\Lambda}
$$

where A denotes the chiral adjoint field, $\mathrm{W}$ is a standard model gauge field strength and $W^{\prime}$ is the field strength of a hidden $\mathrm{U}(1)$ gauge group which is broken at a high scale. This operator is expected to be generated by integrating out heavy messenger fields which are charged under the SM as well as the hidden-sector U(1) gauge group, thus the UV model is a form of gauge mediation [16]. The hidden-sector $\mathrm{U}(1)$ field gets a D-term vev, thus the 
operator becomes a Dirac mass for the SM gaugino, 'marrying' it to the fermionic piece of the chiral adjoint. Defining $D / \Lambda \equiv m_{D}$ we find the operator in equation (3.1) is equivalent to a Dirac mass term $m_{D} \lambda \psi_{A}$ for the gaugino. The chiral multiplet $\mathrm{A}$ is complex, it thus contains both real and imaginary scalar fields which are adjoints under a SM gauge group. The masses of the real and imaginary parts of the scalar adjoint depend greatly on the details of the messenger sector of the model see for example, see for example [17-19]. Many models lead to tachyonic masses for one or the other adjoint, and fixes may produce a great range of adjoint masses. If the scalar adjoints are lighter than the gauginos and sparticles in the theory, then we expect their decays to follow through loops to sets of SM gauge bosons or fermions.

We note that consistent with all symmetries in the theory we may also write the super-potential term

$$
W=\int d^{2} \theta \frac{W_{\alpha}^{Y} W^{\alpha} A}{\Lambda}
$$

where $\mathrm{A}$ is the a chiral SM adjoint field, $W^{Y}$ is the hypercharge field strength and $\mathrm{W}$ is appropriate $\mathrm{SU}(2)$ or $\mathrm{SU}(3)$ field strength tensor. This operator is nearly identical to the 'supersoft' operator which generates Dirac gaugino masses. We may also write

$$
W=\int d^{2} \theta \frac{d^{a b c} W_{3}^{a} W_{3}^{b} S^{c}}{\Lambda^{\prime}}
$$

Where $W_{3}$ is the $\mathrm{SU}(3)$ field strength and $\mathrm{S}$ in the chiral $\mathrm{SU}(3)$ adjoint. Upon integration over $\theta \mathrm{s}$, we see that these superpotential terms produce in the Lagrangian our operators from equations (2.1) and (2.2). These operators are presumably generated much the same way as the supersoft term is, by integrating heavy fields out of the theory as we discuss below. We note that the Lagrangian terms in equation (2.1) and involve the real part of the adjoint. We might also expect similar operators generated by messenger loops to couple imaginary part of A and the SM field strength tensor and dual.

Many aspects of the phenomenology of scalar adjoints resultant from $\mathrm{R}$ symmetric SUSY models have been studied thoroughly [6, 20]. It is known, for example, that an octetgluon-gluon coupling may be generated by integrating out squarks from the $\mathrm{R}$ symmetric theory. Similar diagrams involving squarks and sleptons will generate the the operators in equation (3.2). Squarks and sleptons couple to the real part of the chiral adjoints through Kahler potential D-terms. Due to a cancelation of diagrams, it is known that operators following from sparticle loops are suppressed when left and right sparticle masses are degenerate.

Another source of operators in equations (3.2) and (3.3) are loops of the heavy messenger fields. These messengers carry SM quantum numbers, and in many completions are often various sets of fundamentals and anti-fundamentals under SU(5), see for example $[18,19]$. The relative size of the operators in equations (3.2) and (3.3) will depend on the details of the messenger sector, and on the sparticle spectrum.

Effective dimension 7 operators like those of equation (2.3) may be produced through loops of squarks and sleptons, as these sparticles are charged under multiple SM gauge groups and couple to the Higgs. Thus in 'supersoft' models we find numerous effective 
operators which couple scalar adjoints to pairs of gauge bosons. As a phenomenological note, the real part of the supersoft adjoints may also decay to quarks/leptons through loops. These decays, however, do not always dominate adjoint branching fractions - there are various regions of parameter space where these decays are subdominant to decays into gauge bosons. This region of parameter space will be of special interest to the phenomenological discussions of the next section.

\section{Production and decay of scalar adjoints}

We will now consider the collider phenomenology of our effective operators. We will focus on the phenomenology of colored states as they should be produced with large cross section at the LHC. As such, we will study the production and decay of a single scalar color octet. We will assume that, besides mass and kinetic terms, the octet has only dimension 5 couplings to SM gauge bosons resulting from the operators in equation (2.1). Our simple model has three parameters, the mass of the octet $m_{S}$, and the two effective operator coefficients $\Lambda_{1}$ and $\Lambda_{2}$. In a UV complete model we would know the exact ratio between the operator coefficients, not knowing the details of the high energy model, however, we will consider these two parameters to be independent. The two operator coefficients control the couplings of the scalar color octet to three pairs of SM gauge bosons $g g$ and $g \gamma$ and $g Z$.

We consider here only the single octet production process $p p>S$, which proceeds through gluon fusion. The total production cross section is proportional to the gluongluon decay width, $\Gamma_{g g}$, of the octet, and is given by,

$$
\sigma(p p \rightarrow S)=\Gamma_{g g} \varepsilon \frac{16 \pi^{2}}{s m_{S}} \int_{\frac{m_{S}^{2}}{s}}^{1} \frac{d x}{x} f_{g}(x) f_{g}\left(\frac{m_{S}^{2}}{s x}\right)
$$

where $m_{S}$ is the mass of the scalar, $f_{g}$ are the parton distribution functions (PDFs) for gluons, $x$ is the momentum fraction of the initial gluons, and $\varepsilon=1 / 32$ takes into account the interchange of summed and averaged over states in the decay rate and production cross section. The octet decay width into gluons $\Gamma_{g g}$ is given by

$$
\Gamma_{g g}=\frac{40}{3} \frac{m_{S}^{3}}{32 \pi \Lambda_{2}^{2}} .
$$

We note that the decay width scales like $m_{S}^{3}$ leading to fairly wide resonances for larger masses of the octet.

The production cross section of the octet decreases with increasing parameter $m_{S}$, it also decreases with increasing scale of the effective cut-off $\Lambda_{2}$. Higher order corrections to the production of color-octet scalars can have very large effects. A K-factor is used to scale up the tree level production cross section of the scalar octet. A typical K-factor for the process $g g \rightarrow S$ at $\sqrt{s}=14$ is between 2.4 and 3.6 for a TeV scale octet [21]. In the analyses in this work we choose a K-factor of 2 for octet production at $\sqrt{s}=8 \mathrm{TeV}$ and a $\mathrm{K}$-factor of 2.5 for a sensitivity projection at $\sqrt{s}=14 \mathrm{TeV}$.

In figure 2 we plot the octet production cross section vs. $m_{S}$ for the process $g g \rightarrow S$ in $8 \mathrm{TeV}$ proton-proton collisions. In the figure we have chosen the effective operator 


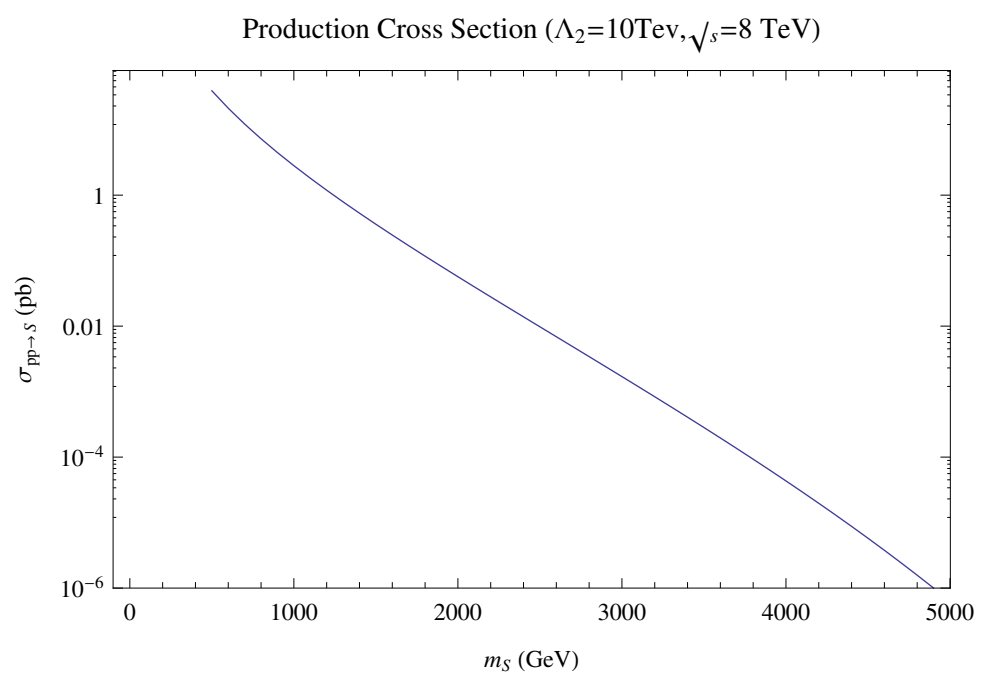

Figure 2. Production cross section of a single scalar octet vs. $m_{S}$ in pp collisions at $\sqrt{s}=8 \mathrm{TeV}$ with $\Lambda_{2}=10 \mathrm{TeV}$. These cross sections were generated with MadGraph 5 .

coefficient $\Lambda_{2}$ to be $10 \mathrm{TeV}$, but this total production cross section may easily be re-scaled for various values of the effective cut-off.

We will now consider the decays of the scalar octet. In our model there are three possible diboson decay channels for the octet, $S \rightarrow g g, g \gamma, g Z$. That is, decays into two gluons, a gluon photon pair, and a gluon $\mathrm{Z}$ pair. Using the effective Lagrangian in equation (2.1), we may calculate the branching fractions. In the limit that the octet is much heavier than the $\mathrm{Z}$ boson, that is $m_{Z} \ll m_{S}$, the branching fractions are given by,

$$
\begin{aligned}
& B R_{g \gamma}=\frac{\Gamma_{g \gamma}}{\Gamma_{g \gamma}+\Gamma_{g g}+\Gamma_{g Z}}=\frac{c_{W}^{2}}{1+\frac{10}{3} \Lambda_{1}^{2} / \Lambda_{2}^{2}} \\
& B R_{g Z}=\frac{\Gamma_{g Z}}{\Gamma_{g \gamma}+\Gamma_{g g}+\Gamma_{g Z}}=\frac{s_{W}^{2}}{1+\frac{10}{3} \Lambda_{1}^{2} / \Lambda_{2}^{2}} \\
& B R_{g g}=\frac{\Gamma_{g g}}{\Gamma_{g \gamma}+\Gamma_{g g}+\Gamma_{g Z}}=\frac{1}{1+\frac{3}{10} \Lambda_{2}^{2} / \Lambda_{1}^{2}}
\end{aligned}
$$

where $s_{W}$ and $c_{W}$ are the sine and cosine of the weak angle and $\Lambda_{i}$ are the effective operator coefficients. The exact form of the branching fractions are given in the appendix. By varying the ratio of effective couplings $\Lambda_{1} / \Lambda_{2}$, we may change the branching fraction rates into the various diboson channels. For generic completions of the effective theory, for example including sets heavy vector-like quarks with electric charge in the range $1 / 3$, we expect the ratio of the branching fractions $\Gamma_{g \gamma} / \Gamma_{g g}$ to be in the percent range. This branching fraction is not insignificant, and together with the large octet production cross section it will mean that there is a substantial signal in the $g \gamma$ resonance channel. As we will see below, this is an interesting and a relatively clean channel, it will therefore be important for scalar octet searches.

Above we have discussed single production of scalar octets however pair production of color octet scalars can occur. The production is dominated by gluon fusion. Due to 
kinematics and p-wave suppression, the pair production cross section falls rapidly with increasing $m_{S}$. Pair production of octets falls below the fb range for LHC energy of $14 \mathrm{TeV}$ when $m_{S} \lesssim 1.5 \mathrm{TeV}$ [8]. Possible signals for pair production are $S S \rightarrow 4 g, 3 g+V, 2 g+V V$ where the $\mathrm{V}$ is a $\gamma$ or $\mathrm{Z}$. Final state topologies will vary depending on the choice of and decay channel for $\mathrm{V}$. In the most realistic models, the branching fraction $S \rightarrow V g$ is in the percent range, as mentioned above, therefore total production and decay into $2 g+V V$ final states will be highly suppressed. However the final process $p p \rightarrow S>V g S>g g$ with two reconstructed octet resonances may be an interesting possible discovery channel for lighter octet masses.

So far we have discussed signals for color octet scalars. However we note that all of our mentioned completions will also contain an $\mathrm{SU}(2)$ adjoint scalars which should couple to pairs gauge bosons due to equivalent new physics. In the case of supersymmetric completions we may naturally evolve not only the dimension 5 operator in equation (2.2), but also the effective dimension 7 operators of equation (2.3), providing a very rich possible phenomenology.

Though in this work we will not carry out a full collider simulation, we may comment on possible event topologies for the discovery of this state. Possible production channels for the $\mathrm{SU}(2)$ triplet scalar, $\mathrm{T}$, involve vector boson fusion, in which the adjoint is produced along with a pair of forward jets $q q \rightarrow j j+T$; and associated production where the adjoint is produced along with an electroweak gauge boson through a quark fusion process, $q q \rightarrow V+T$. For special models where the dimension 7 operators may be non-zero, gluon fusion may also be a possible production channel $g g \rightarrow T$ where the cross section is proportional to $\mathrm{SU}(2)$ breaking Higgs vevs. We expect the $\mathrm{SU}(2)$ adjoint to decay through the dimension 5 couplings to two electroweak vector bosons. This makes for quite interesting final state signals, as we may hope to reconstruct the heavy vector bosons in the decay from pairs of leptons, or, perhaps, as a hadronic "fat-jets". We may also observe a significant number of events with relatively hard photons. This is an interesting topic for further collider studies.

\section{Current limits}

We will now discuss the bounds on parameter space which result from various $8 \mathrm{TeV}$ analyses in Run I of the LHC. In our model, production and decay of the octet produces many interesting final state topologies, therefore there are multiple search channels that can be relevant in the constraint or discovery of the scenario. Both ATLAS and CMS have performed searches for heavy resonances decaying into various final states, and one standard search for new colored resonances is in the dijet resonance channel, $p p \rightarrow X \rightarrow j j$ [22, 23]. Here a heavy state, $X$, is produced and then decays hadronically. These searches constrain the parameter space of our color adjoint operators as the scalar octet generically has significant branching fraction into the gluon-gluon final state. In addition ATLAS and CMS have performed searches for resonances which decay to a photon and jet final state [24, 25]. This search is relevant to our model since the scalar has a decay mode $S \rightarrow g \gamma$. The monojet search $p p \rightarrow j+E_{\mathrm{T}}$ is an important inclusive search channel for constraining new 
physics [26, 27]. This analysis will be relevant to our decay channel $S \rightarrow g Z$, where the $\mathrm{Z}$ decays to missing energy $(Z \rightarrow \nu \nu)$. Finally, CMS has a search for a heavy resonance decaying to a jet and a hadronically tagged vector boson $p p \rightarrow X \rightarrow j+V$ [28]. This analysis will be relevant to our decay channel $S \rightarrow g Z$, where the $\mathrm{Z}$ decays hadronically $(Z \rightarrow j j)$. Below, we will explore the bounds on our parameter space from each of these studies.

\subsection{The jet $+\gamma$ channel}

We first discuss constraints from searches for $\gamma+j$ resonances on the process $g g \rightarrow S \rightarrow \gamma g$. The benchmark models used in these analyses include production of non-thermal quantum black holes $(\mathrm{QBH})$ and excited quarks $\left(q^{*}\right)$, however the search constrains our color octet model as well.

The ATLAS search for photon plus jets used the following search criteria:

- At least one isolated photon with $p_{T}>125 \mathrm{GeV}$.

- At least one hard jet with $p_{T}>125 \mathrm{GeV}$.

- Photon required to have angular separation $\Delta R(\gamma, j)>1.0$ between leading photon and all other jets with $p_{T}>30 \mathrm{GeV}$.

- Photon and jet required to be in the central region of the detector with $\left|\eta_{\gamma}\right|<1.37$ and $\left|\eta_{j}\right|<2.8$.

- Pseudo-rapidity separation between jet and photon of $|\Delta \eta(\gamma, j)|<1.6$.

- Highest $p_{T} \gamma$ and jet candidates used to compute $m_{\gamma j}$, which is binned.

The SM background for this search is dominated by $\gamma+j$ production and QCD dijet production. The SM production of $\gamma+j$ lacks a resonant process and is generated via Compton scattering of quarks and gluons, and through quark-antiquark annihilations. Electroweak processes account for about $1 \%$ of background. The analysis performed by ATLAS observed no excess of events over SM backgrounds, and reported limits on production and decay of their benchmark models to $\gamma j$ final states.

We recast the ATLAS search at $\sqrt{s}=8 \mathrm{TeV}$ with $20.3 \mathrm{fb}^{-1}$ of data in order to rule on the parameter space of our color octet model. We implemented the effective Lagrangian using FeynRules [29], then using MADGRAPH 5 [30] we generated events for the process $g g \rightarrow S \rightarrow \gamma g$ in pp collisions at $8 \mathrm{TeV}$. The events were showered with Pythia [31] and run through the PGS detector simulator [32]. We then implemented the ATLAS cut-based analyses on our data to determine the efficiency of the ATLAS search for our model. A plot of signal efficiency vs $m_{S}$ is given in figure $4 \mathrm{a}$.

In general, we find signal efficiencies of order 50 percent for scalar masses between 1 and and $4 \mathrm{TeV}$, with the efficiency dropping rapidly at low invariant mass.

For each point in parameter space we specify the values of $m_{S}, \Lambda_{1}, \Lambda_{2}$. We then calculate the total number of events which pass the selection criteria, $N_{\text {evt }}=\sigma \times L \times$ $\epsilon \times B R_{g \gamma}$, in each invariant mass bin. In figure 3 we plot the total rate $\sigma \times \epsilon \times B R_{g \gamma}$ 


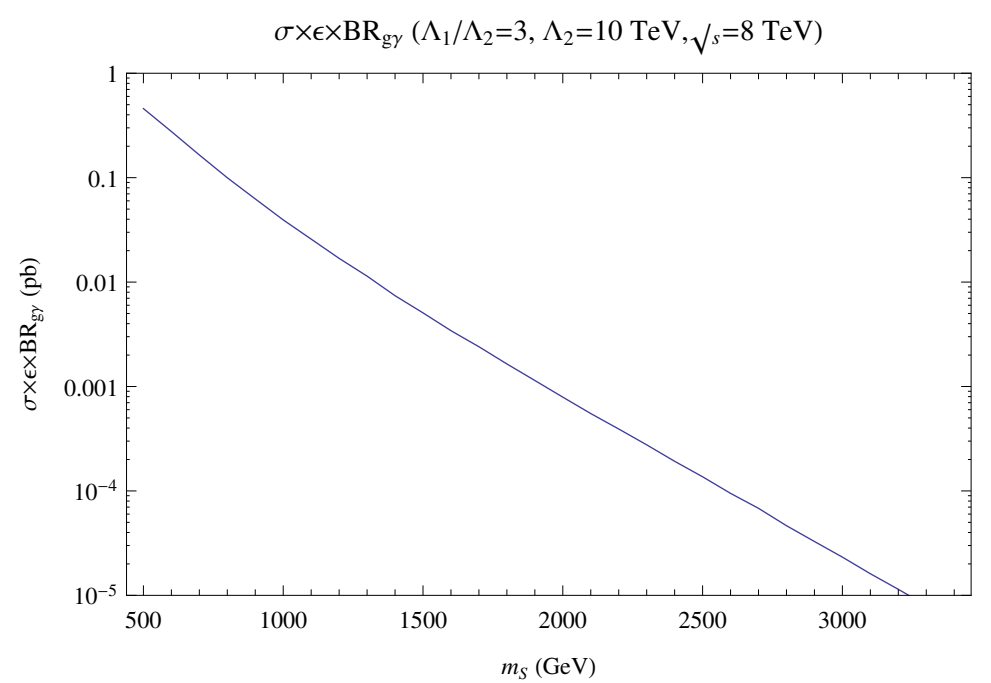

Figure 3. The rate $\sigma \times \epsilon \times B R_{g \gamma}$ of the scalar octet resonance at $8 \mathrm{TeV}$ where $\Lambda_{2}=10 \mathrm{TeV}$ and $\Lambda_{1}=30 \mathrm{TeV}$. This rate includes the efficiency with which events passes the ATLAS selection criteria.

vs. scalar mass $m_{S}$ for the benchmark values $\Lambda_{2}=10 \mathrm{TeV}$ and $\Lambda_{1}=30 \mathrm{TeV}$. At this benchmark point, for octet masses below about $2 \mathrm{TeV}$, the total rate is in the few fb range, and therefore potentially detectable. We show the $95 \%$ confidence level (CL) exclusions in the $\Lambda_{1}-\Lambda_{2}$ plane for various values of the scalar mass in figure 5 .

We see that exclusions become weaker as the scalar mass increases, as is expected due to a rapidly decreasing production cross section. For a given mass, the production rate is set by the parameter $\Lambda_{2}$, with the rate decreasing as $\Lambda_{2}$ increases. The branching fraction into the $\gamma \gamma$ final state is set by the ratio of scales $\Lambda_{1} / \Lambda_{2}$. Thus the total signal rate decreases as this ratio of scales increases. We may exclude effective cut-offs in the 5-10's of $\mathrm{TeV}$ range for $m_{S}$ of the order a few $\mathrm{TeV}$. We note that the effective operator paradigm is not valid to arbitrarily low cut-off scales, but will be valid only when the $\sqrt{s}<2 M_{\text {mess }}$ where $M_{\text {mess }}$ are the mass of the UV messengers integrated out of the theory.

\subsection{Dijet channel}

Dijet searches tightly constrain the existence of new colored resonances. The ATLAS search for dijet resonances was done at $\sqrt{s}=8 \mathrm{TeV}$ with $20.3 \mathrm{fb}^{-1}$ of data. The benchmark models that are considered by ATLAS include excited quarks, QBHs, heavy $W^{\prime}$ gauge fields decaying to quarks, and scalar octet particles decaying to a pair of gluons.

The ATLAS dijet search criteria is as follows:

- At least 2 anti-kt jets with rapidity $|y|<2.8$.

- Two hardest jets (leading and subleading) are required to have $p_{T}>50 \mathrm{GeV}$.

- An invariant mass cut of $m_{j j}>250 \mathrm{GeV}$ is placed on the two hardest jets.

- Separation of rapidities $\frac{1}{2}\left|y_{\text {lead }}-y_{\text {sublead }}\right|<0.6$. 


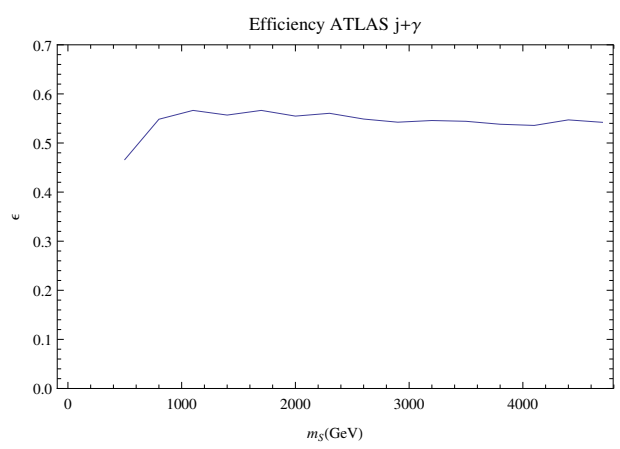

(a)

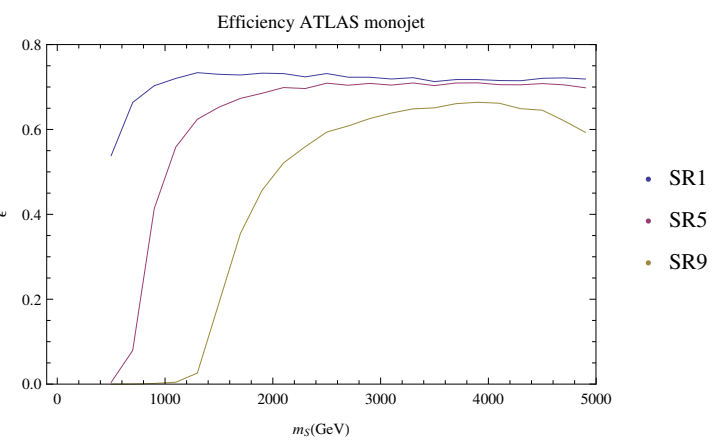

(b)

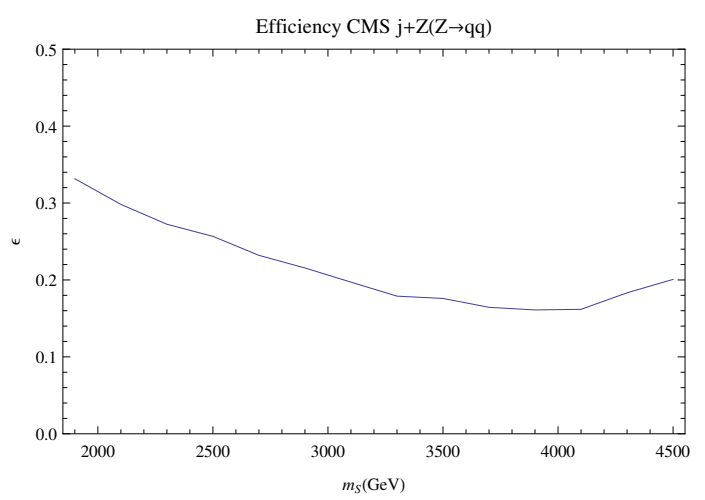

(c)

Figure 4. Efficiency of our simulated events passing the selection criteria in the search channels considered for $\sqrt{s}=8 \mathrm{TeV}$ analyses at the LHC. The upper plots are the signal efficiencies for the ATLAS searches for photon plus jet (left) and monojets (right) corresponding to the decay modes $S \rightarrow g \gamma$ and $S \rightarrow g Z(Z \rightarrow \nu \nu)$ respectively. The lower plot is the kinematic efficiency of the CMS search for hadronically decaying heavy bosons for the decay mode $S \rightarrow g Z(Z \rightarrow q q)$.

SM backgrounds in this channel are dominated by QCD processes with a sub-percent mixture of additional SM processes. The background has a smoothly falling spectrum as the dijet invariant mass of the two highest $p_{T}$ jets (leading and sub-leading), $m_{j j}$ increases [33].

ATLAS analyzed these results to constrain a scalar octet benchmark model. Efficiency cuts for this model range from $61 \%$ to $63 \%$. The ATLAS analysis showed no excess for dijet invariant masses up to $4.5 \mathrm{TeV}$ and placed limits on production rates of their octet. The 95\% CL upper-limit on $\sigma \times A$ with $100 \%$ branching fraction to $g g$ final state was reported by ATLAS. We recast these results to set bounds on the parameter space of model in which the scalar octet has multiple decay channels. By rescaling the ATLAS total rate for the process $g g \rightarrow S \rightarrow g g$ we place bounds in the $\Lambda_{1}-\Lambda_{2}$ plane for various values of $m_{S}$. We show exclusions in figure $5 \mathrm{~b}$.

For a given octet mass $m_{S}$, the total production rate depends on the effective coefficient $\Lambda_{2}$, with the rate decreasing as $\Lambda_{2}$ increases. The total branching fraction of the octet into gluons is then given by the ratio of scales $\Lambda_{1} / \Lambda_{2}$, with the digluon rate increasing as this ratio is increased. We thus expect this search to put the tightest limits on the scalar octet 


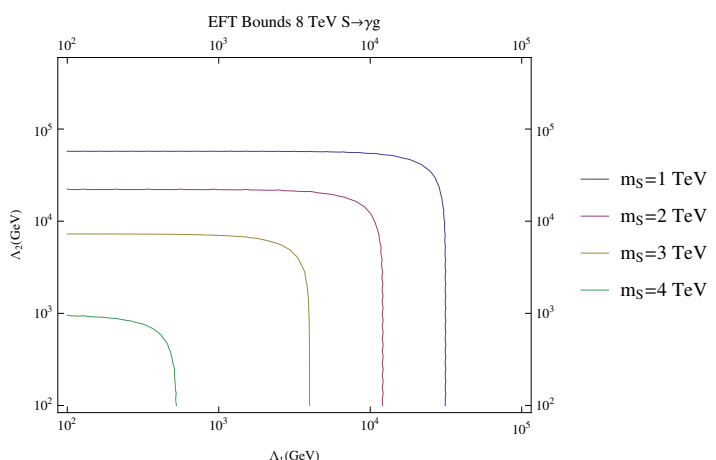

(a)

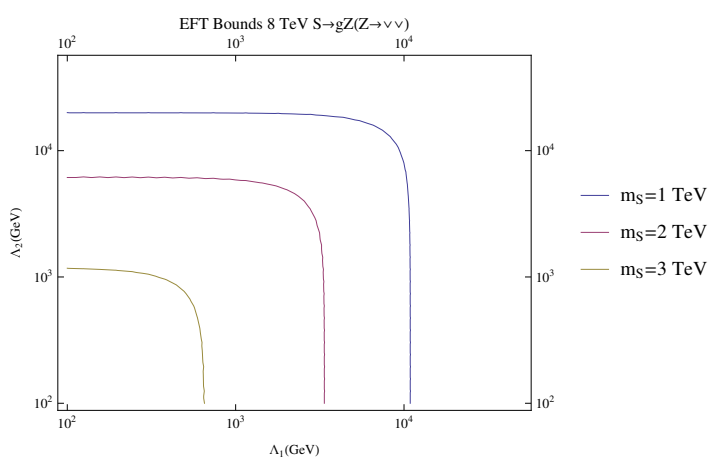

(c)

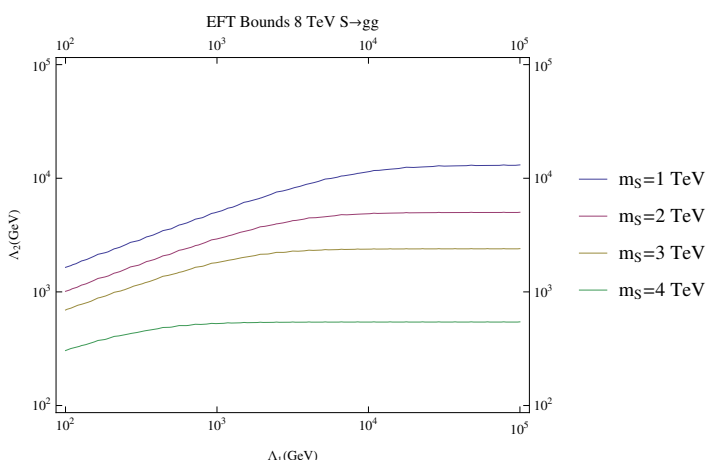

(b)

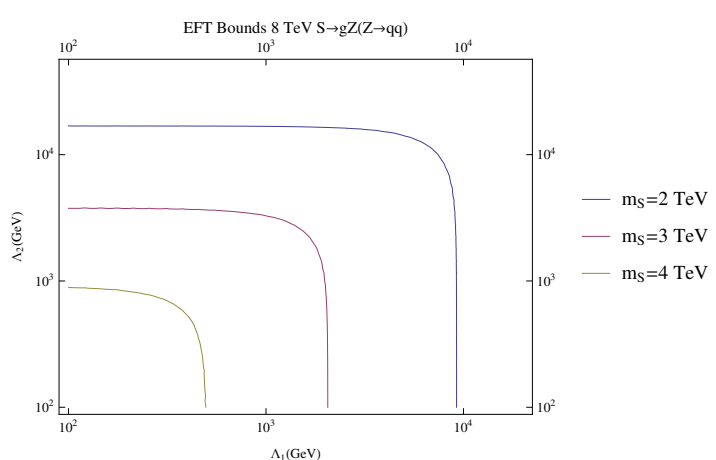

(d)

Figure 5. Bounds on the $\Lambda_{1}-\Lambda_{2}$ for 4 values of the octer mass $m_{S}$. Exclusions follow from 4 LHC $8 \mathrm{TeV}$ analyses. The top left follow from searches in the $p p \rightarrow X \rightarrow g \gamma$ final state. The top right follow from searches in the $p p \rightarrow X \rightarrow j j$ final state. The bottom left follow from searches in the $p p \rightarrow j+E_{\mathrm{T}}$ final state. The bottom right follow from searches in the $p p \rightarrow j+V, V \rightarrow j j$ final state.

model when $\Lambda_{1}$ is large compared to $\Lambda_{2}$, that is, when the effective couplings $S g \gamma$ and $S g Z$ are suppressed.

\subsection{The monojet channel}

The monojet search channel is a standard inclusive analysis that applies to many BSM scenarios. In the model we are considering, the process $g g \rightarrow S \rightarrow Z g$ will lead to a host of interesting signal topologies, among which is a monojet signature. This final state is achieved when the $Z$ decays invisibly. We will now recast the ATLAS $\sqrt{s}=8 \mathrm{TeV}$, $20.3 \mathrm{fb}^{-1}$ monojet analysis to rule on our scenario.

Monojet searches look for an event topology with a hard jet in the central detector region and several hundred GeV of missing energy. The ATLAS monojet search selection criteria is as follows:

- Events required to have $E_{\mathrm{T}}>150 \mathrm{GeV}$ and at least one jet of $p_{T}>30 \mathrm{GeV}$ with $|\eta|<4.5$.

- Leading jet with $p_{T}>120 \mathrm{GeV}$ and $|\eta|<2.0$. 


\begin{tabular}{|c|c|c|c|c|c|c|c|c|c|}
\hline Signal Regions & SR1 & SR2 & SR3 & SR4 & SR5 & SR6 & SR7 & SR8 & SR9 \\
\hline Minimum $\boldsymbol{E}_{\mathrm{T}}(\mathrm{GeV})$ & 150 & 200 & 250 & 300 & 350 & 400 & 500 & 600 & 700 \\
\hline 95\% CL exclusion for $\sigma \times A \times \epsilon(\mathrm{fb})$ & 726 & 194 & 90 & 45 & 21 & 12 & 7.2 & 3.8 & 3.4 \\
\hline
\end{tabular}

Table 1. Signal regions and exclusions from ATLAS monojet search.

- For a monojet like topology, the ratio $p_{T} / E_{\mathrm{T}}>0.5$.

- Separation between leading jet and direction of missing transverse momentum $\Delta \phi\left(j, E_{\mathrm{T}}\right)>1.0$.

- Veto of events with electrons or muons with $p_{T}>7 \mathrm{GeV}$ or isolated tracks with $p_{T}>10 \mathrm{GeV}$ and $|\eta|<2.5$.

Events passing the cuts were divided into 9 signal regions (SR) defined by the minimum amount of $E_{\mathrm{T}}$ in the SR. Limits where 95\% CL limits were placed on $\sigma \times A \times \epsilon$ for the different SR as no excess of events was observed. The signal regions for the ATLAS analysis are shown in the table 1.

The main backgrounds for this search are dominated by $Z(\rightarrow \nu \nu)+$ jets and $W+$ jets production. Smaller contributions come from $Z / \gamma^{*}\left(\rightarrow l^{+} l^{-}\right)+$jets, multijet, $t \bar{t}$, and diboson processes. The search found no excess of monojet events in the $8 \mathrm{TeV}$ dataset.

We utilize this search results to constrain parameter space. We generate events for the process $p p \rightarrow S \rightarrow Z g \rightarrow \nu \nu g$ in MADGRAPH 5. The events are showered with Pythia and then run through the PGS detector simulator. We then implemented the monojet cut based analysis to calculate the efficiency for events in the various signal regions. The calculated efficiency for scalar octets model in various signal regions is found in figure 4b. The overall efficiency of the monojet search for this process is fairly high. We note that our events differ from many models searched for in the monojet channel. For example a cast of DM models in which a gluon is emitted as initial state radiation favor softer gluons with $p_{T}$ peaked at low values. In our events however, the gluon in emitted as a hard decay product in the final state, thus will have a much harder $p_{T}$ which is more amenable to passing a hard $p_{T}$ cut.

We then calculate the total expected event rate and compare to the reported 95\% CL exclusion limits. The exclusions limit results in the $\Lambda_{1}-\Lambda_{2}$ plane can be seen in figure $5 \mathrm{c}$ for various values of the octet mass $m_{S}$. The monojet search does significantly worse at constraining the scalar octet model than the dijet or $\gamma j$ searches. This is in part because the multiplicative effects of the octet branching ratios into the $g Z$ channel and the $\mathrm{Z}$ branching fraction into neutrinos.

As before, the overall production rate of octets decreases with increasing scale $\Lambda_{2}$. The branching fraction into the $g Z$ final state decreases as the ratio $\Lambda_{1} / \Lambda_{2}$ increases. Thus the shape of the exclusion due to this search is similar to that from the $g \gamma$ search. We note that the ratio of $g \gamma$ to $g Z$ events is strictly related by gauge invariance given the form of our operator with coefficient $\Lambda_{1}$, therefore we expect that any signal for scalar octets observed in the $g \gamma$ would eventually produce a signal in the $g Z$ channel as well. 


\section{$5.4 \quad$ Heavy boson plus jet channel}

The CMS collaboration has searched for heavy resonances which decay to a jet and a massive vector boson in the process $p p \rightarrow X \rightarrow j+V$. The search also applies to heavy resonances which decay to two heavy vector bosons. In these searches the vector bosons decay hadronically, $V \rightarrow j j$, and is reconstructed with a hadronic tag. Benchmark models considered for the CMS analysis are excited quarks $\left(q^{*} \rightarrow q W, q^{*} \rightarrow q Z\right)$, Randall-Sundrum gravitons $\left(G_{R S} \rightarrow W W\right)$, and heavy $W^{\prime}$ bosons $\left(W^{\prime} \rightarrow W Z\right)$. The search also applies to our scenario where a heavy scalar octet decays to a gluon and hadronically decaying $\mathrm{Z}$ boson, $g g \rightarrow S \rightarrow Z g, Z \rightarrow j j$.

To identify a jet as being $\mathrm{Z} / \mathrm{W}$-tagged, the CMS analysis used jet pruning techniques, and require the pruned "fat-jet" with to have mass between $70 \mathrm{GeV}<m_{j}<100 \mathrm{GeV}$. The search criteria for the CMS analysis is as follows:

- At least two jets with $p_{T}^{\text {jet }}>30 \mathrm{GeV}$ and $|\eta|<2.5$.

- At least one of the two highest $p_{T}$ jets is required to be vector-boson tagged "fat-jet".

- The pseudorapidity separation between the leading jets $|\Delta \eta(j, j)|<1.3$.

- A cut on the invariant mass of the two highest $p_{T}$ jets of $m_{j j}>890 \mathrm{GeV}$

The dominant source of background for this search stems from multijet production with $t \bar{t}, W+$ jets, and $Z+$ jets contributing less than $2 \%$. Data was binned by the invariant mass of the jet and tagged "fat-jet" $m_{j j}$. No significant excess in data was reported. We may thus use this search to constrain our parameter space.

Using MADGRAPH 5 we simulated parton level events events for the process $g g \rightarrow$ $S \rightarrow Z g, Z \rightarrow j j$. We estimated the search efficiency for these events using the CMS "fatjet"-tagging efficiency and cuts for kinematic efficiency. The tagging efficiency reported by CMS for a single Z-tagged "fat-jet" ranges from roughly $45 \%$ to $30 \%$, with the Z-tag doing better at lower invariant mass. In our analysis we took the Z-tag jet efficiency to be $40 \%$. In figure $4 \mathrm{c}$ we plot the search efficiency vs octet mass $m_{S}$ in the $\mathrm{Z}+\mathrm{j}$ resonant final state.

Using the production cross section, branching fraction and efficiency for our events, we may then calculate the total number of expected events in each invariant mass bin for each point in our parameter space. We then compare to $95 \%$ CL upper bounds in each mass bin from the CMS search to constrain the parameters. Exclusions are shown in the $\Lambda_{1}-\Lambda_{2}$ plane for various scalar masses $m_{S}$ in figure $5 \mathrm{~d}$. The shape of the exclusion line follows from previous discussions of the $g \gamma$ and $g Z$ final states.

\subsection{Combined results}

Combined results for all of the final state channels under consideration are displayed in figure 6. In the figure we have fixed the scale $\Lambda_{2}$ to be $10 \mathrm{TeV}$, and the ratio $\Lambda_{1} / \Lambda_{2}$ to be 3. This benchmark point is representative of a class of a UV completions with vector-like 


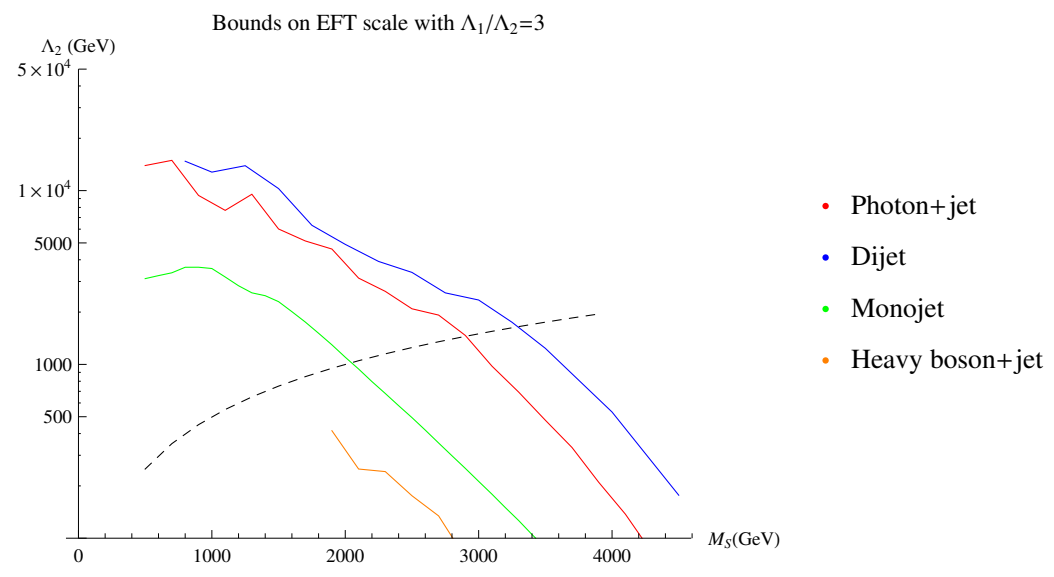

Figure 6. Combined 95\% confidence level bound on EFT scale from all the channels considered for the choice $\Lambda_{2}=.1 \Lambda_{1}$. The black dashed line corresponds to where the validity of the EFT framework breaks down $m_{S}>2 \Lambda_{i}$.

messengers in the multi-TeV range. ${ }^{1}$ Effective cutoffs may be excluded between a few and $10 \mathrm{~s}$ of $\mathrm{TeV}$ over the octet mass range. We see that for high $m_{S}$ regions the dijet search is the most exclusive, while the $\gamma+j$ search is the most exclusive for the low $m_{S}$ regions. While the branching fraction of the octet into $\gamma+j$ is in the percent range for this benchmark point, the background for this channel is quite low. Thus we find that in some of the parameter space of the theory, searches in the $\gamma+j$ channel may be competitive with dijet searches. Due to a very large background, the dijet channel signal efficiency for very light octets is quite low. We might expect that in searches for scalar octets with masses in the 100 s of $\mathrm{GeV}$ range, $\gamma+j$ will generally be the most powerful.

We will now discuss the limits of the effective operator paradigm. The effective operators which we have been studying are generated by integrating out some heavy messenger particles which couple to the scalar octet and to gauge bosons. We know that the effective operator treatment is invalid for scales of the effective cut-off that are below the center of mass energy of the process, that is where the messenger fields are very light. In this low cut-off regime we cannot use the effective terms to accurately calculate the octet production cross section [34]. However, we are assuming that if the octet prefers to decay via loop level couplings, it must have no tree level decays to messenger fields. That is, the mass of the octet must be less than twice the messenger mass $m_{S}<2 M_{\text {mess }}$ and thus the messengers must be offshell in the process. Gladly, we note that whenever this offshell condition, $\sqrt{s}<2 M_{\text {mess }}$, is satisfied, that is exactly the region where the EFT is valid as well. Since the effective operator coefficients are about equal to the UV messenger masses, may thus place a viability condition on the effective operator treatment that the coefficients should satisfy $m_{S}<2 \Lambda$. In figure 6 we have drawn the line of effective operator viability, operator coefficients too light compared to the octet mass are inviable.

\footnotetext{
${ }^{1}$ We implemented a model in Feynrules containing a set of heavy vector-like quarks with qunatum numbers $(3,1)_{2 / 3}$ and a scalar octet. The model was exported to Madgraph NLO to calculate branching fractions of the octet. Resultant branching fractions confirm the benchmark ratio of effective coefficients.
} 


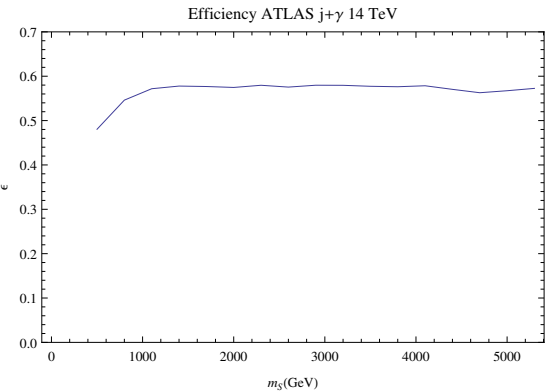

(a)

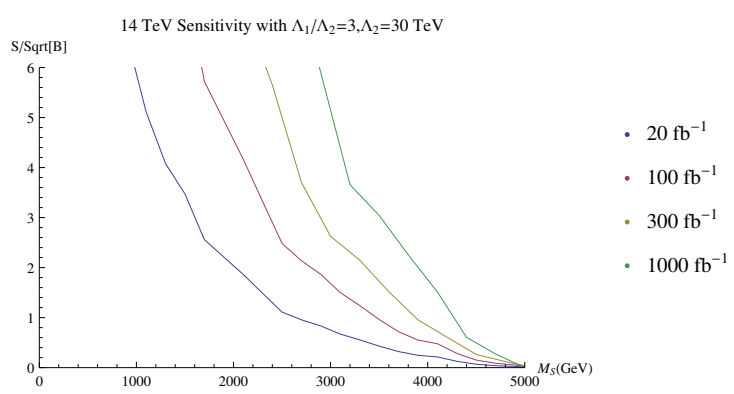

(b)

Figure 7. a.) On the left is the efficiency of signal events for $S \rightarrow g \gamma$ which pass the selection criteria at $14 \mathrm{TeV}$ center of mass energy. b.) On the right is the projected sensitivity for the $14 \mathrm{TeV}$ run in the photon plus jet resonance channel.

\section{Projection for $\mathrm{LHC} 14$}

We will explore the reach of the $14 \mathrm{TeV}$ run of LHC into our parameter space. Below we produce a sensitivity study for the process $g g \rightarrow S \rightarrow g \gamma$ in pp collisions at $\sqrt{s}=14 \mathrm{TeV}$. This search is based on the ATLAS $8 \mathrm{TeV} j \gamma$ resonance search. In this analysis will follow the same cuts as the $8 \mathrm{TeV}$ ATLAS analysis above,

- At least one isolated photon with $p_{T}>125 \mathrm{GeV}$.

- At least one hard jet with $p_{T}>125 \mathrm{GeV}$.

- Photon required to have angular separation $\Delta R(\gamma, j)>1.0$ between leading photon and all other jets with $p_{T}>30 \mathrm{GeV}$.

- Photon and jet required to be in the central region of the detector with $\left|\eta_{\gamma}\right|<1.37$ and $\left|\eta_{j}\right|<2.8$.

- Pseudo-rapidity separation between jet and photon of $|\Delta \eta(\gamma, j)|<1.6$.

- Highest $p_{T} \gamma$ and jet candidates used to compute $m_{\gamma j}$, which is binned.

Our model is implemented in Feynrules. For each point in our parameter space we generate signal events for our process using Madgraph5. We shower the events with Pythia and run them through the detector simulator PGS. We then run a cut based the analysis on the events to determine the search efficiency. In figure 7a we show a plot of signal efficiency vs octet mass $m_{S}$. In general we find efficiencies similar to those of the $8 \mathrm{TeV}$ analysis. We may then calculate the total number of events $N_{\text {evt }}=\sigma \times L \times \epsilon \times B R_{g \gamma}$ in each invariant mass bin.

As in the $8 \mathrm{TeV}$ search, the SM background for this process is dominated by $\gamma+j$ events. In order to estimate the background at $14 \mathrm{TeV}$ we scale up the four parameter fit used to model the SM background in ATLAS's $8 \mathrm{TeV}$ analysis.

We use a simple criteria for estimating sensitivity to new physics. The signal to root background ratio is denoted $S / \sqrt{B}$ where $S$ and $B$ are the expected number of signal and 


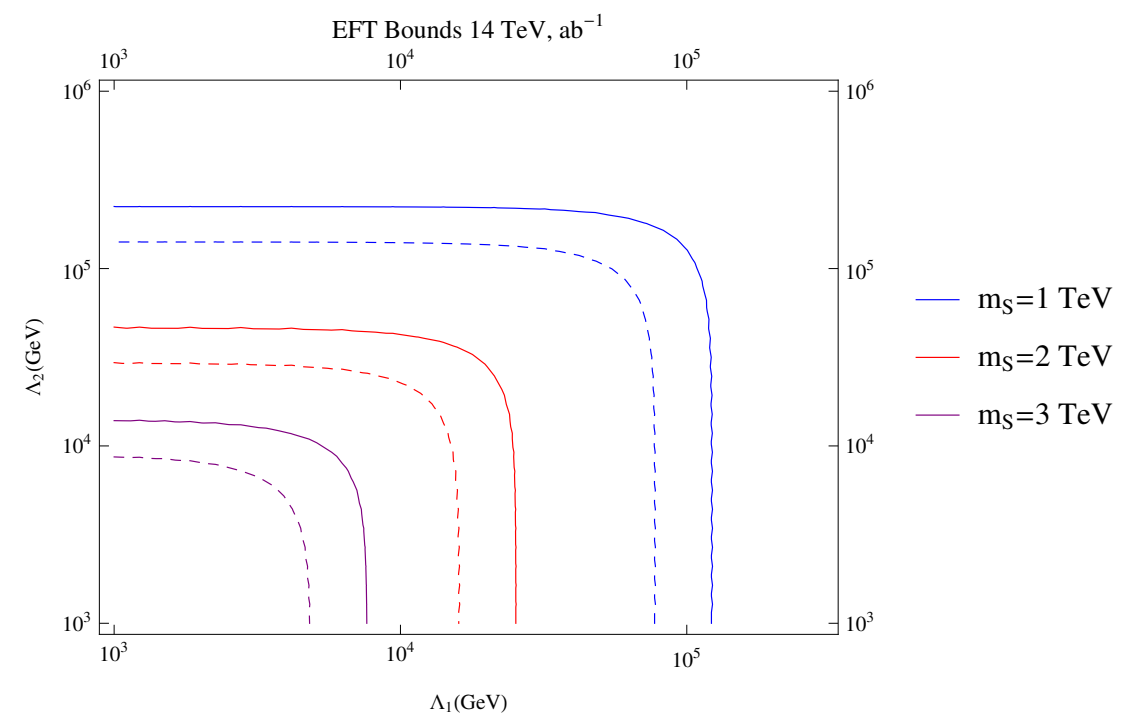

Figure 8. Projected sensitivity of color octet searches in the $g \gamma$ channel for the $14 \mathrm{TeV}$ LHC run. Shown are reached for various valued of the octet mass in the $\Lambda_{2}-\Lambda_{1}$ plane. Solid lines indicate the 2 sigma reach while dotted lines indicate 5 sigma reach.

background events respectively. For Poisson statistics, a search is sensitive to the existence of a new physics if $S / \sqrt{B} \geq 2$ and has discovery potential if $S / \sqrt{B} \geq 5$.

We plot the sensitivity for the $14 \mathrm{TeV}$ process $g g \rightarrow S \rightarrow g \gamma$ in figure $7 \mathrm{~b}$. In the plot we fixed the benchmark effective operator coefficients $\Lambda_{2}=30 \mathrm{TeV}$ and $\Lambda_{1}=90 \mathrm{TeV}$, and then plotted the expected $S / \sqrt{B}$ ratio vs. the octet mass $m_{S}$. We have shown the sensitivity for various values of the run luminosity. For this benckmark point, we find that a octets of $m_{S} \lesssim 1.8 \mathrm{TeV}$ have 5 sigma discovery potential in $100 \mathrm{fb}^{-1}$ of luminosity. In 1 $\mathrm{ab}^{-1}$ octets of $m_{S} \lesssim 3 \mathrm{TeV}$ have discovery potential in this channel. In figure 8 we show the reach in the $\Lambda_{1}-\Lambda_{2}$ plane for various values of the octet mass.

\section{Conclusions}

We have considered a set of effective dimension 5 operators through which standard model adjoint scalars couple to pairs of SM gauge bosons. These operators may present themselves in a variety of models. We have considered supersymmetric and non-supersymmetric UV completions for these scenarios including the popular set of 'R-symmetric' SUSY models, which demand the existence of scalar fields which are adjoints under the SM gauge groups. These operators open up new phenomenological possibilities in the search for scalar adjoint states.

In this work we study the phenomenology of the production and decay of a single color octet scalar which couples to pairs of SM gauge bosons, $g g, Z \gamma, Z g$. We have calculated bounds on this scenario from several analyses from the $8 \mathrm{TeV}$ run of LHC. We have placed lower limits on effective operator coefficients for a variety of octet masses using $\gamma+$ jet, dijet, 
monojet and $\mathrm{V}+\mathrm{j}$ search channels. We have also explored the discovery potential of the octet scalar in the gluon-photon final state for the $14 \mathrm{TeV}$ run of LHC.

These operators open the possibility for a variety of new production and decay channels for scalar octet searches. For example, the decays of the octet scalar in the $\mathrm{Z} g$ channel alone include such final state topologies as jet plus a hard dilepton pair, as well as jets plus $E_{\mathrm{T}}$, and jet+hadronic tagged $\mathrm{Z}$ topologies. Though the branching fraction into leptons is small, this may be an interesting low background channel to search in for new states. Similarly interesting decay channels exist for equivalent operators pertaining to $\mathrm{SU}(2)$ adjoint scalars. We also note that we have here considered only single scalar octet production through gluon fusion. There are, however, stranger production channels to be studied. For example, even if the Sgg coupling were somehow small, the $S g \gamma$ coupling would allow S production through the processes $g g>g^{*}>S \gamma$ and even $g g>g^{*}>S Z$. Models with such adjoints have a variety of collider topologies which would be an interesting topic of further study.

As discussed in the section on high energy models, the dimension 7 operators listed in equation (2.3). are produced at loop level by integrating out heavy squarks/sleptons in R-symmetric SUSY models. This set of operators presents more possibilities for phenomenological study if the operators are not too suppressed. For example once Higgs vevs are inserted, the operators allow production of the $\mathrm{SU}(2)$ adjoint though gluon fusion. The decay of this adjoint may have a striking leptonic signature. More complex scenarios which involve SM adjoints may have even more striking signatures. For example, in the MonoharWise model there exists scalar color octets which are fundamentals and anti-fundamentals of $\mathrm{SU}(2)$. Effective couplings of these adjoints to pairs of vector bosons may lead to very interesting decay chains.

Finally we note that UV completions of our models predict correlations of signals across multiple channels. A combinations of final state searches will be the most powerful tool to rule on the parameter space of these models. This will also help to determine to the correct UV physics if a scalar adjoint signal is seen in a channel. For example, in an R-symmetric-MSSM scenario the detection of a scalar color octet in the dijet resonance channel would demand a specific rate in the $\mathrm{j}+$ photon channel as well. Measuring these rate would give valuable information about the messenger and squark sectors of the theory.

\section{A Additional color-adjoint operators}

For the Monohar Wise model, a color octet Scalar is also an $\mathrm{SU}(2)$ doublet $S_{u}=(8,2)_{1}$, $S_{d}=(8, \overline{2})_{-1}[3]$. We may write the most general terms effective Lagrangian which couples these fields to pairs of SM gauge bosons,

$$
\begin{aligned}
L= & \frac{1}{\Lambda_{g g 1}^{2}} d^{a b c} H^{\dagger} S_{u a} G_{b}^{\mu \nu} G_{c}^{\mu \nu}+\frac{1}{\Lambda_{g g 2}^{2}} d^{a b c} H S_{d a} G_{b}^{\mu \nu} G_{c}^{\mu \nu}+\frac{1}{\Lambda_{g b 1}^{2}} H^{\dagger} S_{u}^{a} G_{a}^{\mu \nu} B^{\mu \nu} \\
& +\frac{1}{\Lambda_{g b 2}^{2}} H S_{d} G_{a}^{\mu \nu} B^{\mu \nu}+\frac{1}{\Lambda_{g w 1}^{2}}\left[H^{\dagger} W^{\mu \nu} S_{u}\right] G_{a}^{\mu \nu}+\frac{1}{\Lambda_{g w 2}^{2}}\left[H W^{\mu \nu} S_{d}\right] G_{a}^{\mu \nu}
\end{aligned}
$$

Were $G^{\mu \nu}$ is the $\mathrm{SU}(3)$ field strength tensor, $W^{\mu \nu}$ is the $\mathrm{SU}(2)$ field strength tensor and $B^{\mu \nu}$ is the $\mathrm{U}(1)$ fields strength tensor. In the first 4 terms $\mathrm{SU}(2)$ indices of the scalar 
octets are contracted with those of the Higgs. In the last two operators $\mathrm{SU}(2)$ indices are contracted between the Higgs, scalar octet and $W^{\mu \nu}$ as indicated by brackets.

Finally we may consider the effective Lagrangian of a bi-adjoint under SU(3) and SU(2)

$$
L=\frac{1}{\Lambda_{s g w}} S_{a}^{j} G_{a}^{\mu \nu} W_{\mu \nu}^{i}+\frac{1}{\Lambda_{s g b}^{3}}\left[H^{\dagger} S_{a}^{j} H\right] G_{a}^{\mu \nu} B_{\mu \nu}^{i}+\frac{1}{\Lambda_{s g g}^{3}}\left[H^{\dagger} S_{a}^{j} H\right] G_{a}^{\mu \nu} G_{\mu \nu}^{i} .
$$

The exact branching fractions for a scalar color octet into gg, $g \gamma$ and gZ final states following from equation (2.1) are,

$$
\begin{aligned}
B R_{g \gamma} & =\frac{c_{W}^{2}}{c_{W}^{2}+s_{W}^{2}\left(1-m_{Z}^{2} / m_{S}^{2}\right)^{3}+\frac{10}{3} \Lambda_{1}^{2} / \Lambda_{2}^{2}} \\
B R_{g Z} & =\frac{s_{W}^{2}\left(1-m_{Z}^{2} / m_{S}^{2}\right)^{3}}{c_{W}^{2}+s_{W}^{2}\left(1-m_{Z}^{2} / m_{S}^{2}\right)^{3}+\frac{10}{3} \Lambda_{1}^{2} / \Lambda_{2}^{2}} \\
B R_{g g} & =\frac{1}{1+\frac{3}{10} \Lambda_{2}^{2} / \Lambda_{1}^{2} c_{W}^{2}+\frac{3}{10} \Lambda_{2}^{2} / \Lambda_{1}^{2} s_{W}^{2}\left(1-m_{Z}^{2} / m_{S}^{2}\right)^{3}},
\end{aligned}
$$

where $c_{W}$ and $s_{W}$ are the cosine and sine of the weak angle.

\section{Acknowledgments}

This work was made possible by fund from DOE grant DE-SC001352.

Open Access. This article is distributed under the terms of the Creative Commons Attribution License (CC-BY 4.0), which permits any use, distribution and reproduction in any medium, provided the original author(s) and source are credited.

\section{References}

[1] P.J. Fox, A.E. Nelson and N. Weiner, Dirac gaugino masses and supersoft supersymmetry breaking, JHEP 08 (2002) 035 [hep-ph/0206096] [INSPIRE].

[2] L.J. Hall and L. Randall, U(1)-R symmetric supersymmetry, Nucl. Phys. B 352 (1991) 289 [INSPIRE].

[3] A.V. Manohar and M.B. Wise, Flavor changing neutral currents, an extended scalar sector and the Higgs production rate at the CERN LHC, Phys. Rev. D 74 (2006) 035009 [hep-ph/0606172] [INSPIRE].

[4] L. Randall and R. Sundrum, A Large mass hierarchy from a small extra dimension, Phys. Rev. Lett. 83 (1999) 3370 [hep-ph/9905221] [INSPIRE].

[5] T. Han, I. Lewis and Z. Liu, Colored Resonant Signals at the LHC: Largest Rate and Simplest Topology, JHEP 12 (2010) 085 [arXiv: 1010.4309] [INSPIRE].

[6] T. Plehn and T.M.P. Tait, Seeking Sgluons, J. Phys. G 36 (2009) 075001 [arXiv:0810.3919] [INSPIRE].

[7] S.Y. Choi, J. Kalinowski, J.M. Kim and E. Popenda, Scalar gluons and Dirac gluinos at the LHC, Acta Phys. Polon. B 40 (2009) 2913 [arXiv:0911.1951] [InSPIRE]. 
[8] C.-Y. Chen, A. Freitas, T. Han and K.S.M. Lee, Heavy Color-Octet Particles at the LHC, JHEP 05 (2015) 135 [arXiv: 1410.8113] [INSPIRE].

[9] L.M. Carpenter and S. Mantry, Color-Octet, Electroweak-Doublet Scalars and the CDF Dijet Anomaly, Phys. Lett. B 703 (2011) 479 [arXiv:1104.5528] [INSPIRE].

[10] D. Carmi, A. Falkowski, E. Kuflik, T. Volansky and J. Zupan, Higgs After the Discovery: A Status Report, JHEP 10 (2012) 196 [arXiv:1207.1718] [InSPIRE].

[11] J. Goodman, M. Ibe, A. Rajaraman, W. Shepherd, T.M.P. Tait and H.B. Yu, Constraints on Light Majorana dark Matter from Colliders, Phys. Lett. B 695 (2011) 185 [arXiv: 1005.1286] [INSPIRE].

[12] A. Mendez and A. Pomarol, One loop induced $H^{+} W^{+} Z$ vertex in the minimal supersymmetry model, Nucl. Phys. B 349 (1991) 369 [INSPIRE].

[13] A. Arhrib, R. Benbrik and M. Chabab, Charged Higgs bosons decays $H^{ \pm} \rightarrow W^{ \pm}(\gamma, Z)$ revisited, J. Phys. G 34 (2007) 907 [hep-ph/0607182] [InSPIRE].

[14] S. Dimopoulos, S. Raby and G.L. Kane, Experimental Predictions from Technicolor Theories, Nucl. Phys. B 182 (1981) 77 [inSPIRE].

[15] C.T. Hill and E.H. Simmons, Strong dynamics and electroweak symmetry breaking, Phys. Rept. 381 (2003) 235 [Erratum ibid. 390 (2004) 553] [hep-ph/0203079] [INSPIRE].

[16] M. Dine, A.E. Nelson, Y. Nir and Y. Shirman, New tools for low-energy dynamical supersymmetry breaking, Phys. Rev. D 53 (1996) 2658 [hep-ph/9507378] [INSPIRE].

[17] L.M. Carpenter, Dirac Gauginos, Negative Supertraces and Gauge Mediation, JHEP 09 (2012) 102 [arXiv: 1007.0017] [INSPIRE].

[18] L.M. Carpenter and J. Goodman, New Calculations in Dirac Gaugino Models: Operators, Expansions and Effects, JHEP 07 (2015) 107 [arXiv: 1501.05653] [INSPIRE].

[19] C. Csáki, J. Goodman, R. Pavesi and Y. Shirman, The $m_{D}-b_{M}$ problem of Dirac gauginos and its solutions, Phys. Rev. D 89 (2014) 055005 [arXiv:1310.4504] [InSPIRE].

[20] S.Y. Choi, M. Drees, J. Kalinowski, J.M. Kim, E. Popenda and P.M. Zerwas, Color-Octet Scalars of $N=2$ Supersymmetry at the LHC, Phys. Lett. B 672 (2009) 246 [arXiv: 0812.3586] [INSPIRE].

[21] A. Idilbi, C. Kim and T. Mehen, Factorization and resummation for single color-octet scalar production at the LHC, Phys. Rev. D 79 (2009) 114016 [arXiv:0903.3668] [INSPIRE].

[22] ATLAS collaboration, Search for new phenomena in the dijet mass distribution using pp collision data at $\sqrt{s}=8 \mathrm{TeV}$ with the ATLAS detector, Phys. Rev. D 91 (2015) 052007 [arXiv: 1407.1376] [INSPIRE].

[23] CMS collaboration, Search for resonances and quantum black holes using dijet mass spectra in proton-proton collisions at $\sqrt{s}=8 \mathrm{TeV}$, Phys. Rev. D 91 (2015) 052009 [arXiv: 1501.04198] [INSPIRE].

[24] ATLAS collaboration, Search for new phenomena in photon+jet events collected in proton-proton collisions at $\sqrt{s}=8 \mathrm{TeV}$ with the ATLAS detector, Phys. Lett. B 728 (2014) 562 [arXiv: 1309.3230] [INSPIRE].

[25] CMS collaboration, Search for excited quarks in the $\gamma+$ jet final state in proton-proton collisions at $\sqrt{s}=8 \mathrm{TeV}$, Phys. Lett. B 738 (2014) 274 [arXiv:1406.5171] [InSPIRE]. 
[26] ATLAS collaboration, Search for new phenomena in final states with an energetic jet and large missing transverse momentum in pp collisions at $\sqrt{s}=8 \mathrm{TeV}$ with the ATLAS detector, Eur. Phys. J. C 75 (2015) 299 [Erratum ibid. C 75 (2015) 408] [arXiv:1502.01518] [INSPIRE].

[27] CMS collaboration, Search for dark matter, extra dimensions and unparticles in monojet events in proton-proton collisions at $\sqrt{s}=8$ TeV, Eur. Phys. J. C 75 (2015) 235 [arXiv: 1408.3583] [INSPIRE].

[28] CMS collaboration, Search for massive resonances in dijet systems containing jets tagged as $W$ or $Z$ boson decays in pp collisions at $\sqrt{s}=8$ TeV, JHEP 08 (2014) 173 [arXiv: 1405.1994] [INSPIRE].

[29] A. Alloul, N.D. Christensen, C. Degrande, C. Duhr and B. Fuks, FeynRules 2.0 - A complete toolbox for tree-level phenomenology, Comput. Phys. Commun. 185 (2014) 2250 [arXiv: 1310.1921] [INSPIRE].

[30] J. Alwall, M. Herquet, F. Maltoni, O. Mattelaer and T. Stelzer, MadGraph 5: Going Beyond, JHEP 06 (2011) 128 [arXiv:1106.0522] [INSPIRE].

[31] T. Sjöstrand, S. Mrenna and P.Z. Skands, PYTHIA 6.4 Physics and Manual, JHEP 05 (2006) 026 [hep-ph/0603175] [INSPIRE].

[32] pythia/pgs package for MadGraph, https://launchpad.net/pythia-pgs-for-mg.

[33] R.M. Harris and K. Kousouris, Searches for Dijet Resonances at Hadron Colliders, Int. J. Mod. Phys. A 26 (2011) 5005 [arXiv:1110.5302] [INSPIRE].

[34] G. Busoni, A. De Simone, J. Gramling, E. Morgante and A. Riotto, On the Validity of the Effective Field Theory for Dark Matter Searches at the LHC, Part II: Complete Analysis for the s-channel, JCAP 06 (2014) 060 [arXiv:1402.1275] [INSPIRE]. 